Aus der $k \mathrm{k}$. dermatologischen Klinik des Prof. F. J. Piok in Prag.

\title{
Ueber das Lymphangioma cutis cysticum circumscriptum.
}

\author{
Von \\ Docent Dr. Ludwig Waelseh, \\ I. Assistent der Klinik. \\ (Hierzu Tafel IX u. X.)
}

Am 4. December 1898 wurde unserer Klinik die Patientin K. M., 26jährige Taglöhnerin, von der chirurgischen Klinik des Herrn Professor Wölfle $\mathbf{r}$ wegen einer Affection an der Haut der rechten Thoraxseite übersendet.

Die Kranke war am 4. November 1898 mit einer Struma parenchymatosa in die obige Klinik aufgenommen worden. Die Affection, wegen der sie uns zugeschickt wurde, bestand angeblich seit frühester Jugend, ohne sich zu vergrössern. Die Struma bestand seit dem 15. Lebensjahre und hatte sich allmälig aus kleinen haselnussgrossen Knoten am Halse entwickelt. Ziemlich kurze Zeit nachher, als dieser Tumor zu wachsen begonnen, trat Heiserkeit auf, die, in wechselnder 1ntensität, die ganze Zeit besteht. Ausserdern ist Patientin in der letzten Zeit sehr dyspnoisch, hat keine Schlingbeschwerden.

Die Untersuchung der Patientin ergab mässigen Exophthalmus und eine unregelmässig conturirte Schwellung des Halses (Umfang $41 \mathrm{Cm}$.) Die letztere rührt her von mehreren knollenförmigen Strumaknoten, die hauptsächlich auf der rechten Halsseite gelegen sind. Die Haut über den derben Knoten, sowie die letzteren selbst sind vollständig verschieblich.

An der Haut der rechten Thoraxseite, in der Höhe der 5. Rippe, von der binteren Axillarlinie bis zur Mamillarlinie reichend, fand sich ein circa $5 \mathrm{Cm}$. langer, $1 \mathrm{Cm}$. breiter Streifen, der sich nach den beiden Enden hin verschmächtigte und aus lauter Bläschen zusammensetzte, welche, dicht beieinander stehend, einen serösen Inhalt aufwiesen, der hie und da hämorrhagisch getrübt war. In den mittleren Theilen des Herdes waren diese Bläschen flach, wenig über das Niveau der Haut erhaben, klein, an der Peripherie dagegen rundlich, kugelig hervortretend, gross, mehrkämmerig. Beim Anstechen entleerte sich der klare Inhalt 
Waelsch.

dieser grösseren Bläschen theilweise unter gleichzeitigem Collabiren eines Theiles derselben, zeigte alkalische Reaction, mikroskopisch spärliche Lymphkörperchen; bei tieferem Einstechen entleerte sich der ganze Inhalt unter geringem Nachsickern von Blut. Zwischen den einzelnen Bläschengruppen zeigten sich ectasirte Gefässreiserchen.

Parallel diesem streifenförmigen Gebilde fand sich ein zweiter, schräg verlaufender Streifen, der sich ausschliesslich, wie Taf. IX zeigt, aus erweiterten Blutgefässen zusammensetzte und seiner ganzen Configuration nach als ein Naevus teleangiectaticus angesprochen werden musste. Die als blaurothe Streifen und Reiser hervortretenden Gefässchen entleerten sich unter Fingerdruck, um nach Aufhören des Druckes sich langsam wieder za füllen.

Dieser untere Streifen stand mit dem oberen durch ein schräg verlaufendes Band in Verbindung, welches sich aus lauter kleinen, in die Haut eingelagerten, derb anzufühlenden Bläschen zusammensetzt. Gegen die Mamma zu standen kleine Gruppen von Bläschen isolirt in die Haut eingelagert.

Einen Monat nach der ersten Untersuchung wurde uns die Patientin wieder zugeschickt. Sie war inzwischen am 20.|XII. 1898 operirt worden, indem, wie ich aus der mir freundlichst von Herrn Prof. Wölfler zur Einsicht überlassenen Krankengeschichte entnahm, das auf der linken Seite bis unter die Clavicula reichende Kropflager exstirpirt wurde, während der rechte grosse Knoten mit dem Thermocauter an verschiedenen Stellen gestichelt wurde.

Wir waren nun ungemein überrascht über die Veränderung, welche die Affection an der Brust aufwies. In der Nachbarschaft des oberen Streifens waren allenthalben frische Bläschen aufgeschossen. (Es widersprach dies der Angabe der Patientin, dass der Tumor seit der Jugend nicht gewachsen sei. Nachdem die Patientin eine sehr geringe Intelligenz zeigte, hatten wir schon von allem Anfange an diese ibre Angabe mit einem gewissen Misstrauen aufgenommen, das durch die jetzt erfolgte Beobachtung sich als begründet erwies.) Auch das vorerwähnte Haemangiom zeigte auffallende Veränderungen. Es war bis auf spärliche Gefässreiserchen vollständig geschwunden und an seiner Stelle und in genau derselben Ausdehnung und Configuration, welche es gelegentlich der ersten Untersuchung aufgewiesen und die durch die Abbildung festgehalten worden, waren zahlreiche Bläschen desselben Ausehens wie die oben beschriebenen, a ufgeschossen. 
Wir stellten auf Grund des klinischen Bildes, des Befundes von kleinen Hohlräumen, die mit Lymphe gefüllt waren und welche sehr zahlreich an der Haut, sogar unter unseren Augen sich gebildet hatten, die Diagnose eines Lymphangioma cysticum, und die histologische Untersuchung ergab die Richtigkeit dieser Annahme.

Zum Zwecke der histologischen Untersuchung wurde der Patientin ein grösseres Hautstückchen entnommen, das sowohl einen Theil des reinen Lymphangioms enthielt, als auch jener Hautpartie entstammte, auf welcher es, auf Boden des ursprünglichen Haemangioms, zur Entstehung desLymphangioms gekommen war. Die excidirten Hautstückchen wurden in Alkohol, Sublimat, Zenker'scher Flüssigkeit fixirt.

Die histologische Untersuchung ergab einen verschiedenen Befund je nachdem die Schnitte von der Peripherie der Affection, wo der Process erst im Beginne seiner Entstehung begriffen war, oder von den weiter entwickelten oder endlich von den auf der Höhe der Entwicklung stehenden Herden herstammte.

An Schnitten, welche die Peripherie trafen, wo sich makroskopisch absolut keine sichtbaren pathologischen Hautveränderungen constatiren liessen, bestanden Veränderungen an den Blutgefässcapillaren und zwar hauptsächlich an denen des subpapillaren Netzes, weniger an denen der Papillen. Die Capillaren waren etwas erweitert, von Rundzellen dicht umgeben, und zeigten ausserdem Wucherungen ihrer Wandelemente. Zwischen den Rundzellen liessen sich, manchmal gehäuft, mit goldgelbem Pigment angefüllte Zellen nachweisen, die sich in spärlicher Zabl auch sonst allenthalben im Bindegewebe fanden. Dort, wo die kleinen Capillaren quer getroffen waren, liess sich eine geringe Erweiterung derselben sowie der die Gefässchen umgebende Rundzellenmantel deutlich constatiren. Dieses Rundzelleninfiltrat bestand ausschliesslich um die Gefässchen, nur hie und da fand es sich in umschriebenen Anhäufungen, die scheinbar ohne Verbindung mit den Blutgefässen waren. Bei genauerem Zusehen liess sich aber auch hier ein kleines Gefässchen erkennen, an welches diese Herde gebunden waren. Im Deckepithel und dessen Anbängen, im Bindegewebe zeigten sich sonst keine pathologischen Veränderungen. 
Bei Schnitten, welche mehr gegen die makroskopisch sichtbaren Hautveränderungen geführt waren, liessen sich im Bindegewebe spaltförmige oder ovale Hohlräume erkennen, die von einer geronnenen Flüssigkeit erfüllt waren und wandständig einkernige Zellen, in kleinen Haufen beisammen liegend, aufwiesen. Diese Hohlräume hatten nun entweder vollständig glatte Contour, oder aber sie zeigten kleine, in die Spitze zulaufende Ausbuchtungen, die, mehr weniger weit, in das benachbarte Bindegewebe reichend, ebenso wie das Lumeu der Hohlräume selbst, von einem zarten Endothel ausgekleidet waren. Von der Spitze dieser Fortsätze liess sich dann sehr oft ein feiner Faden verfolgen, der sich aus, der Länge nach an einander gereibten, endotheläbnlichen Zellen zusammensetzte und in das Bindegewebe sich hinein erstreckte. Das Endothel liess sich fast an allen Hohlräumen und hier zumeist in continuirlicher Lage mit seinen schma'en, spindelförmigen Kernen deutlich nachweisen. An manchen Stellen sprangen die Kerne etwas stärker buckelförmig gegen das Lumen vor und waren dann entsprechend kürzer. Es mangelte dieses Endothel den Hohlräumen nur an jenen Stellen, an welchen, wie bei den später noch zu beschreibenden, grossen, blasenartigen Gebilden, besonders an deren Basis, das Bindegewebe förmlich auseinander gedrängt und gezerrt war, in Folge des starken Druckes und der Expansion der in den Blasen vorhandenen Flüssigkeit. Ferner fehlte es manchmal an jenen Stellen, an welchem ihm, entweder von innen oder von aussen, im Lumen oder im Bindegewebe, Rundzellen angelagert waren.

Diese kleinen Hohlräume fanden sich zumeist in den Papillen oder im Bindegewebe, knapp uuter den Retezapfen, und drängten dann in dem Masse, als sie sich vergrösserten, auch die tiefer gelegenen Bindegewebsschichten auseinander. In diesen letzteren liessen sich Hohlräume nur noch in einer Höhe constatiren, welche ungefähr der der Talgdrüsen entsprach. Sie waren hier ungemein klein, spaltförmig, zumeist der Hautoberfläche parallel laufend.

Ausserdem fanden sich auch pathologische Veränderungen in dem, diesen Erweiterungen benachbarten Bindegewebe, u. zw. in Form eines kleinzelligen Infiltrates, das in umschriebenen. 
kleineren und grösseren Herden, jedoch immer ziemlich dicht, allenthalben in der Nachbarschaft derselben sich constatiren liess. Dort, wo diese entzündlichen Veränderungen fehlten, zeigte sich aber das benachbarte Bindegewebe nicht normal, indem es nämlich, ungemein kernreich, eine gewisse Verdichtung erkennen liess. Es fanden sich auch bier, jedoch bedeutend spärlicher, die Rundzellen der vorerwähnten Infiltrate, und nachdem sich bei den frischen Infiltrationen überall eine allmälige Umwandlung des Rundzellenkernes in einen spindeligen Kern erkennen liess und unter Zurücktreten der Zahl der Leucocyten die Zahl der Spindelzellen im Bereiche dieser Herde mehr und mehr zunahm, so ist wohl der Schluss gestattet, dass das vorerwäbnte verdichtete und kernreiche Bindegewebe eben diesen seinen Zellenreichthum der vorausgegangenen Entzündung, einer Metamorphisirung der Rundzellen in Spindelzellen verdankt.

In dem Masse, als dann die Zahl und Grösse dieser Hohlräume mehr und mehr zunahm, rückten sie immer näher an einander, rareficirten das zwischen ihnen befindliche Bindegewebe, so dass endlich schmale Septen aus an einander gepressten Bindegewebszügen sie von einander trennten. Auch gegen das Epithel zu vergrösserten sie sich auf Kosten des zwischen ihnen und dem Epithel befindlichen Bindegewebes, das sich dann nur mehr in Form eines schmalen Streifens nachweisen liess.

Durch diese Vergrösserung und den dadurch bewirkten, von unten her ausgeübten Druck, kam es zu einer fast vollständigen Abflachung und Ausgleichung der Papillen und Retezapfen unter gleichzeitiger Verschmächtigung des Deckepithels, so dass über die grössten und sehr dicht an einander gelagerten, kleineren Efflorescenzen dasselbe in einer Flucht, in reducirter Zellenschichte hinüberzog. Ausserdem wurde durch diesen von unten her ausgeübten Druck auch das über den Hohlräumen befindliche Bindegewebe mit dem Deckepithel, nach oben bin, nach der Seite des geringeren Widerstandes, vorgedrängt, so dass kleine, polsterartige Erhebungen oder geradezu bläschenartige Efflorescenzen zu stande kamen.

Bei diesen grossen Efflorescenzen, welche klinisch als von Serum erfuilte Bläschen imponirten, zeigte das histologische Bild grosse, blasigeHohlräume, die augenscheinlich einerDehiscenz der, die klei- 
neren Hohlräume von einander trennenden Septa ihre Entstehung verdankten. Dafür sprach der Rest dieser Septa, der, wie es Török treffend bezeichnet, wie ein Sporn gegen das Lumen vorragte; es war dann aus einer mebrkämmerigen eine einkämmerige Blase entstanden. Auch hier zeigten sich noch deutlich Entzündungserscheinungen, besonders an der dem Corium zugewendeten Fläche.

Jedoch nicht nur auf diese Weise, durch die Ausweitung eines vorhandenen Hohlraumes unter gleichzeitiger Vermehrung seiner Wandelemente der Fläche nach, liess sich das Entstehen der grösseren Bläschen erklären, sondern es zeigten sich auch allenthalben an den Spalten und Höhlen Veränderungen, die darauf hinwiesen, dass eine Neubildung ron Lymphgefässen stattfand.

Ich habe schon oben erwähnt, dass sich an den erweiterten Spalten kleine Endothel-Fortsätze constatiren liessen, die sich in ihrem Anfangsstadium als zarte Endothelreiser darstellten, welche sich in das Bindegewebe erstreckten. Man konnte nun aber deutlich erkennen, dass an der Stelle, wo diese Sprossung stattfand, das rundliche oder spaltförmige Lumen wie in eine Spitze ausgezogen war, als deren Ausläufer der Endothelspross in das Bindegewebe hineindrang. Diese Spitze weitete sich immer mehr und mehr, sowohl der Länge als der Breite nach aus und liess dann aus sich ein feines Lymphgefässstämmchen entstehen, das mit dem grösseren Hohlraum communicirend, von zartem, neugebildeten Endothel ausgekleidet war. In Folge dieser Neubildung von Lymphgefässen durch Sprossung kam es auch oft dazu, dass benachbarte Hohlräume mit einander in Communication versetzt wurden Die successie Erweiterung dieser neugebildeten Lymphgefässe und Aufnahme ihrer Wandungen in das ursprüngliche grössere Lumen, sowie die Erweiterung des Verbindungsganges zweier benachbarter Hohlräume spielte bei dem Zustandekommen der grossen bläschenartigen Gebilde ebenfalls mit.

Neben dieserNeubildung vonLymphgefässen durch Sprossung liess sich aber auch ein anderer Entstehungsmodus derselben constatiren. Es fanden sich nämlich in den umschriebenen Anhäufungen von Rundzellen im Bindegewebe sowie auch in den vorerwähnten verdichteten Bindegewebspartien ungemein 
feine Spalträume, die von endothelartigen Zellen mit spindeligen Kernen begrenzt, eine Verbindung mit Lymphspalten oder Lymphräumen nur undeutlich erkennen liessen.

Die erwähnten spalt- oder bläschenförmigen Lymphräume waren in sich ganz geschlossen. Eine Communication derselben mit tieferen Lymphgefässen liess sich nirgends mit Sicherheit nachweisen, dagegen fanden sich an der unteren Wand der Hohlräume in einigen Schnitten erweiterte Lymphgefässstämmchen, die schräg oder senkrecht aufsteigend und sich allmälig verschmächtigend, in eine feine Spitze auslaufend, an die untere Wand anstiessen. An dieser Stelle fand sich in der Nachbarschaft kleinzelliges Infiltrat, häufiger Bindegewebswucherung, so dass es. törmlich den Eindruck machte, dass durch diese entzündlichen Veränderungen eine Abknickung oder Verlegung des abführenden tieferen Lymphgefässstämmchens zustande gekommen war.

Im Gegensatze zu den hier nicht besonders ausgesprochenen Veränderungen an den Blutgefässen liess jenes Hautstückchen, das von der Hautpartie stammte, an welcher vor dem Auftreten des Lymphangioms ein Haemangiom bestanden hatte, auch bedeutende Veränderungen an den Blutgefässcapillaren constatiren. Es fanden sich hier dieselben in verschiedenen Stadien der Erweiterung, u. zw. waren es hauptsächlich die des subpapillaren Gefässnetzes. Von der kleinen, gerade noch als Erweiterung kenntlichen Veränderung fanden sich alle Uebergänge bis zu grossen rundlichen oder unregelmässigen Hohlräumen, das Lumen immer vollgefüllt mit rothen Blutkörperchen. In den Papillen liessen sich deutlich die oben beschriebenen Veränderungen der Lymphgefässe constatiren, nur war es an manchen Stellen zu einem Riss in dem das Lymphgefäss von dem Blutgefäss trennenden Bindegewebe gekommen und infolge dessen zu einer Haemorrhagie in das. Lymphangiom, die ja auch schon klinisch an manchen Stellen der bläschenförmigen Efflorescenzen durch Verfärbung des Inhaltes. kenntlich war.

Sonstige pathologische Veränderungen an der Hant betrafen nur noch die elastischen Fasern. Dieselben zeigten sich dort, wo entzündliche Veränderungen in der Nachbarschaft des Lymphangioms bestanden, etwas rareficirt und fehlten fast voll- 
ständig, an manchen Stellen auch total, dort, wo es zu den oben beschriebenen Verdichtungen des Bindegewebes gekommen war. Ebenso fanden sie sich auch nur in einzelnen Fäserchen, in jenen schmalen Bindegewebsschichten, die die Lymphgefässerweiterungen von dem Deckepithel trennten.

Endlich liessen sich Proliferationsvorgänge an den Haarbälgen constatiren. Dieselben zeigten deutlich Wucherungen des Epithels wit Bildung seitlicher Knospen. Die Talgdrüsen erwiesen sich als vollständig normal, manchmal liess sich zwischen ihrer membrana propria und dem derselben anliegenden Bindegewebe ein schmaler, endothelbekleideter Lymphraum constatieren.

Es ergab also die histologische Untersuchung Befunde, die in Uebereinstimmung mit den von $\mathrm{Schmidt,} \mathrm{Török,}$ Freudweiler, Pawlow erhobenen stehen und sich von diesen nur unterschieden durch das in unserem Falle stärker Ausgesprochensein der entzündlichen Veränderungen in der Nachbarschaft der wuchernden Blut- und Lymphgefässe, ein Moment, auf das ich aus später noch zu erörternden Gründen ganz besonderes Gewicht legen möchte.

Hatten wir es nun im folgenden Falle zu thun mit einer Lymphangiektasie, mit dem Producte einer Lymphstauung oder aber mit einem echten Lymphangiom, einer Neubildung von Lymphgefässen? Es ist dies eine Frage, welche immer bei Erörterung derartiger Fälle gestellt wurde, jedoch bisher keine zufriedenstellende Beantwortung fand. Ich möchte im Folgenden die Möglichkeit der Entstehung auf die eine oder die andere Weise erörtern.

Was die Entstehung dieser Tumoren durch Neubildung betrifft, so stimme ich mit Langhans, Esmarch und Kulenkampf, Nasse und Schmidt darin überein, dass die Ursache dieser Geschwulstbildung in localen Gewebsveränderungen gelegen sein muss, denn die Lymphstauung allein kann unmöglich zu solchen „umschriebenen“ Lymphgefässerweiterungen Veranlassung geben.

Anderseits können wir aber von einer Lymphstauung absolut nicht absehen, nachdem ja, wie Schmidt treffend hervorhebt, die Vergrösserung eines vorhandenen oder gebildeten 
Raumes ohne Druck von Innen nicht zu erklären ist. Dieser Druck kann durch nichts anderes als durch Stauung bedingt sein.

Die oben erwähnten localen Gewebsveränderungen rerlegt nun Nasse in die embryonale Entwicklungszeit. Zur Erklärung des Modus dieser Fntstehung können drei Ansichten herangezogen werden, die von Rindfleisch, von Wegner, endlich von Billroth, Virchow, Winniwarter und Weichselbaum.

Rindfle isch sucht die Ursache darin, dass durch Umwandlung jungen Bindegewebes in festes Gewebe in der Umgebung bestehender Hohlräume Retraction stattfindet, wodurch dieselben erweitert werden. Wegner nimmt die Möglichkeit einer homäoplastischen Bildung der Lymphgefässe von den Endothelien der ursprünglichen Lymphgefässe an, während die letztgenannten Autoren die Entstehung neuer Lymphgelässe aus jungen Bindegewebszellen, eine heteroplastische Entstehung, als möglich binstellen.

Ich möchte nun auf Grund meiner Präparate mich dahin aussprechen, dass alle drei erwähnten Arten von Entwicklungsstörungen bei der Entstehung der Lymphangiome mitspielen, aber nicht nur zur Zeit der embryonalen Entwicklung, sondern auch während des späteren Lebens. Dafür spricht das Wachsthum dieser angeborenen Gebilde, welche wir an unserem Falle beobachten konnten und das Fre udweile $\mathbf{r}$ bei seinem in langer Beobachtung stehenden Falle in ausgezeichneter Weise beschrieben hat. Das Primäre bei der Entstehung dieser Geschwülste ist jedenfalls eine Wucherung der Lymphgefässendothelien, für die wir eine Erklärung abzugeben nicht in der Lage sind, die in einem "Ueberschuss an productiver Kraft dieser Zellenelemente gelegen sein muss, welche sie zu quantitativ abnormen Leistungen befähigt". Für diese homöoplastische Bildung der Lymphgefässe durch Sprossung sprechen neben meinen Befunden auch die Beobachtungen von Wegner, Nasse, Török, Freudweiler, Pawlow.

Dabei möchte ich aber auch die Möglichkeit einer heteroplastischen Entstehung, im Sinne der oben erwähnten Autoren nicht von der Hand weisen, nachdem ich Befunde, wie sie Nasse und Schmidt beobachteten, Neubildung von Lymph- 
gefässen im Bindegewtbe, ohne deutliche Communication mit bestehenden Lymphräumen, ebenfalls verzeichnen konnte.

Und was endlich das ersterwähnte Moment betrifft, so möchte ich auch die Wahrscheinlichkeit der Entstehung der Lymphgefässerweiterung im Sinne Rindfleisch's anvehmen. Dafür spricht das jugendliche, kernreiche Bindegewebe, das im Gefolge der Entzündung um die wuchernden Lymphgefässe herum sich entwickelt hat und die im Anschluss daran sich entwickelnde Verdichtung des Bindegewebes, das ich oben beschrieben.

Durch die erwähnten entzündlichen Veränderungen, welche durch das Wachsthum der Lymphgefässe förmlich provocirt werden und durch den Ausgang derselben kommt es, wie ich an mehreren Stellen sehen konnte, zu einer Abknickung, manchmal sogar Verlegung der abführenden Lymphgefässästchen, so dass, wenn auch, wie $\mathrm{N}$ asse hervorhebt, zahlreiche Anastomosen zwischen den kleinsten Lymphgefässen bestehen, doch eine Behinderung der Abfuhr der Lymphe sich ergibt, vorausgesetzt, dass sich dieselben an einem grösseren Hautteritorium abspielen, wie dies bei unserem Falle gewesen.

Dass hier locale Veränderungen vor sich gegangen, die einen günstigen Boden abgaben für die Entstehung localer Stauung, dafür spricht auch das Vorhandensein des Haemangioms, auf dem dann die Lymphangiomefflorescenzen aufschossen. Es steht dies in Uebereinstimmung mit der Ansicht Un n a's, dass jede Lymphangiektasie ein doppeltes Hindernis voraussetzt, auf der renösen und auf der lymphatischen Bahn. Ein Hindernis für den Abfluss der Lymphe, das nur die Lymphgefässe betrifft, kann eine Lymphangiektasie nicht erzeugen, weil die Lymphe dadurch gar nicht gestaut würde, indem noch freier Abfluss derselben durch die Venen möglich ist.

Ich möchte hier noch die Anschauung Besnier's streifen, der die Fälle von Schmidt und Török nicht als reines Lymphangioma ansieht, sondern dieselben für Haemangiome hält, deren Inhalt eine "seröse Transformation" erfahren.

Dem widerspricht aber der histologische und klinische Betund. Denn erstens ergab die Untersuchung der ganz frischen 
Efflorescenzen an der Peripherie niemals das Bild eines Haemangioms, indem die erweiterten Räume sich immer von Lymplee mit Lymphkörperchen erfuillt zeigten, und andererseits schossen ja unter unseren Augen frische Efflorescenzen auf dem Boden des bestehenden Haemangioms auf, die sich ebenfalls a]s nicht mit Blut gefüllt erwiesen. Man könnte vielleicht den Einwand erheben, dass gerade dieser unser Fall beweisend für die Besnier'sche Anschauung ist, und das mit Recht, wenn thatsächlich das Haemangiom verschwunden und aus ihm ein Lymphangiom entstanden wäre. Dagegen spricht aber die mikroskopische Untersuchung des Hautstückchens dieser Stelle. Es fanden sich nämlich dort, wo makroskopisch von dem Haemangiom gar nichts mehr zu sehen war, im mikroskopischen Bilde deutliche Gefässektasien, die aber durch die dichte Aneinanderlagerung der über ihnen entstandenen Lymphgefässerweiterungen im klinischen Bilde vollständig verdeckt worden waren

Ich möchte nach dem soeben Auseinandergesetzten die vorliegende Neubildung, nachdem die Lymphgefässwucherung das Primäre ist, als echtes Lymphangiom bezeichnen, jedoch auch darauf hinweisen, dass die Lymphstauung bei der Entstehung und dem Wachsthum dieser Geschwülste eine wichtige Rolle spielt. 


\section{Erklärung der Abbiläungen auf Taf. IX u. $\mathbf{X}$.}

Taf. I. Aufnahme der Kranken Mitte December 1898.

Taf. II. Aufnahme der Kranken Mitte Januar 1899.

\section{Literatur.}

1. Török L. \& Noyes: Lymphangioma circumscriptum, Monatshefte für prakt. Dermatologie, 1890 , Bd. II.

2. Schmidt. Beiträge zur Kenntniss der Lymphangioma. Archiv für Dermatologie und Syphilis, 1890, pag. 529.

3. Freudweiler. Lymphangioma circumscriptum s. cystoides cutis. Archiv für Dermatologie und Syphilis, 1897, Bd. XLI.

4. Langhans, citirt bei Nase.

5. Esmarch \& Kulenkampf, citirt bei Nasse.

6. Nasse. Archiv für klinische Chirurgie, XXXVIII, 1888.

7. Rindfleis ch. Lehrbuch der pathol. Histologie, Leipzig, 1886, pag. 149 .

8. Wegner. Ueber Lymphangioma. Langenbeck's Archiv, 1876, Band XX.

9. Virchow's Archiv VII.

10. Billroth. Beiträge zur patholog. Histologie, Berlin, 1858.

11. Winiwarter, Langenbeck's Archiv XII.

12. Weichselbaum. Virchow's Archir LVIV.

13. Unna. Histopathologie der Hautkrankheiten, pag. 932.

14. Besnier \& Doyon. Pathologie et traitement des maladies de la pean par Kaposi, 1891, Bd. II.

15. Pawlow. Ein Fall von Lymphangioma circumscriptum der Eaut. Monatshefte f. prakt. Dermatologie. Bd. XXIX. 


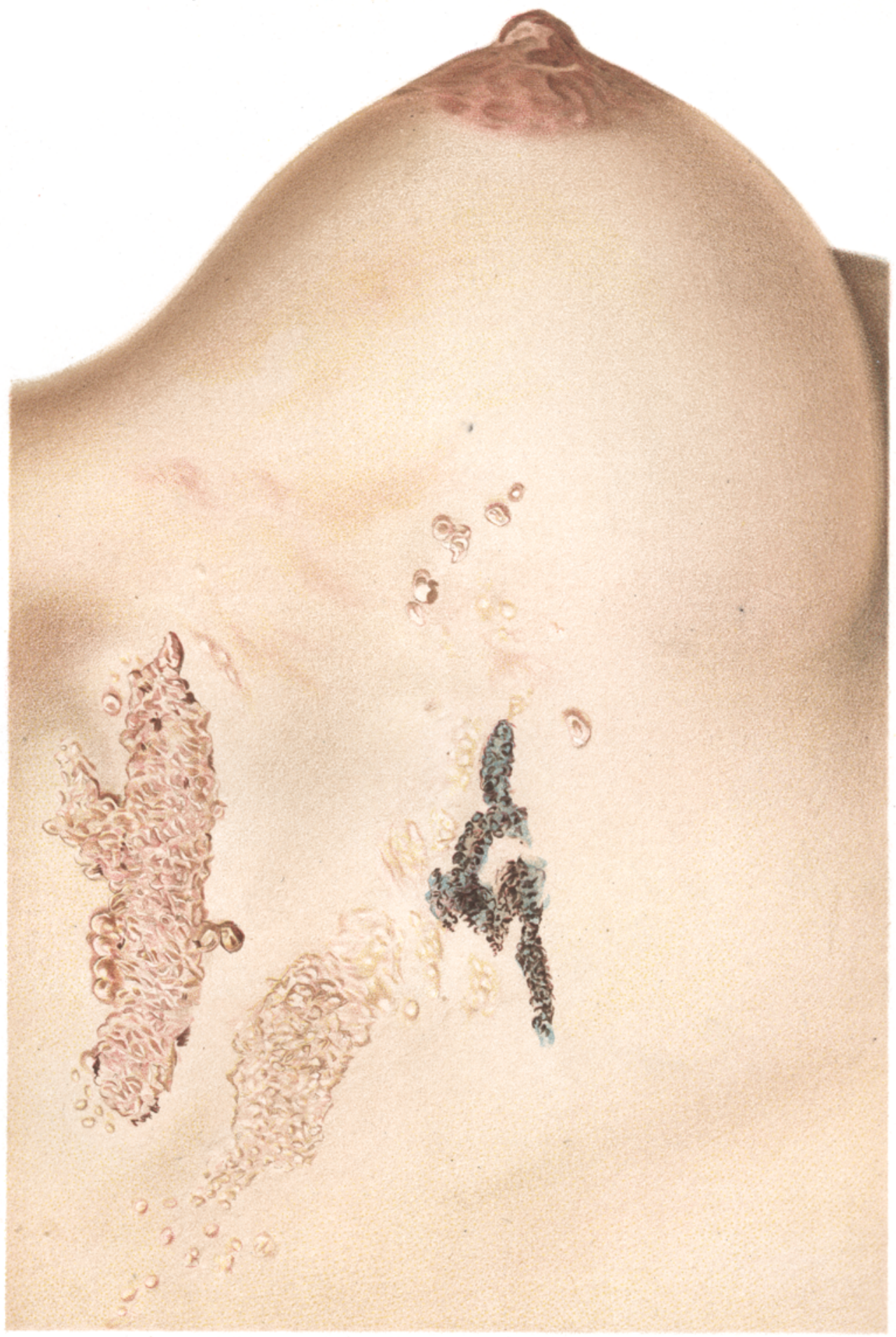




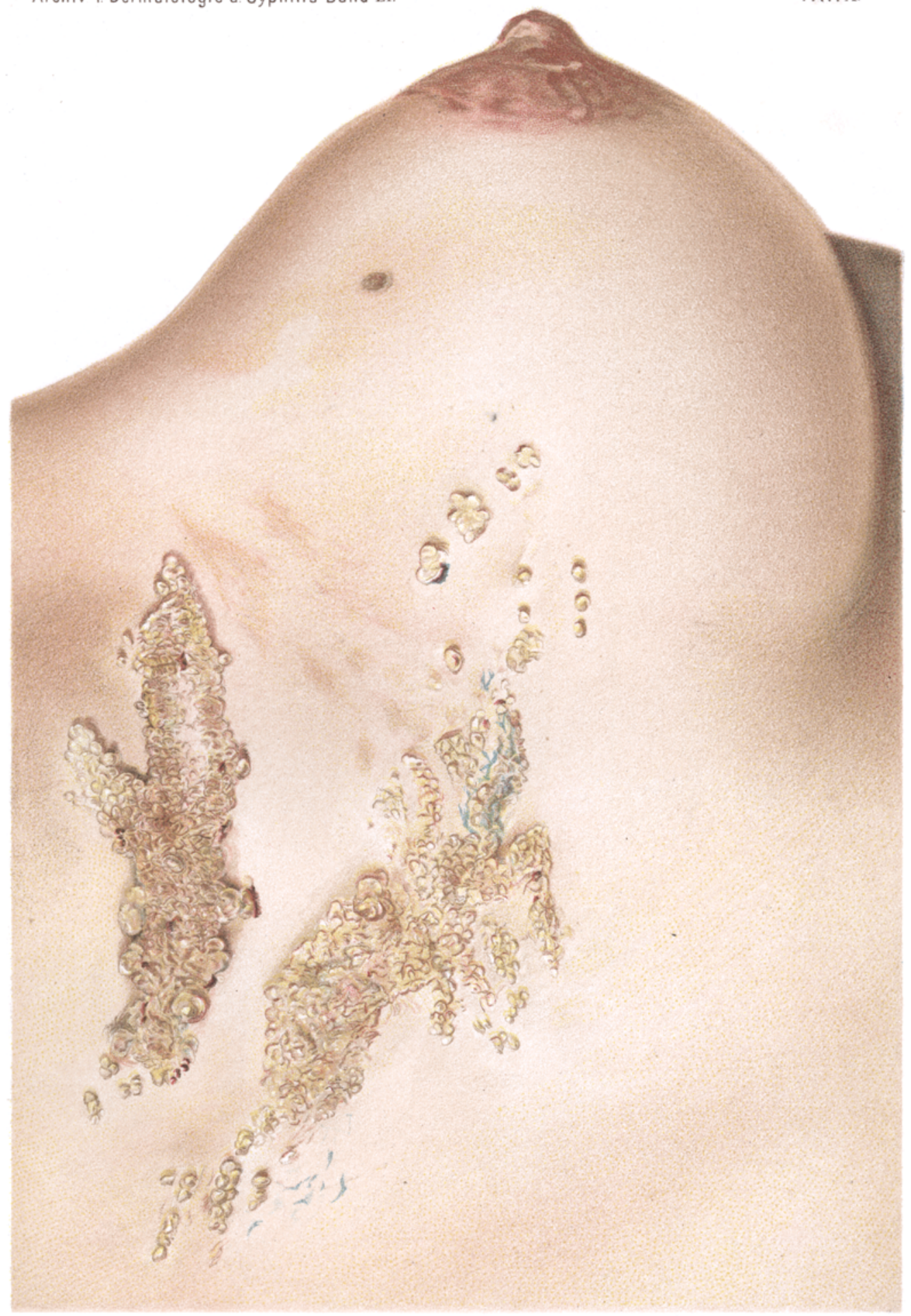

